\title{
Cofactor NAD(P)H Regeneration: How Selective Are the Reactions?
}

\author{
Joseph W.H. Burnett, ${ }^{1}$ Russell F. Howe, ${ }^{2}$ and Xiaodong Wang ${ }^{1,3, *}$
}

${ }^{1}$ Chemical and Materials Engineering, School of Engineering, University of Aberdeen, Aberdeen AB24 3UE, United Kingdom

${ }^{2}$ Chemistry Department, University of Aberdeen, Aberdeen AB24 3UE, United Kingdom

${ }^{3}$ Department of Engineering, Lancaster University, Lancaster LA1 4YW, United Kingdom

*Correspondence: xiaodong.wang@lancaster.ac.uk (X. Wang)

\begin{abstract}
The regeneration of $\mathrm{NAD}(\mathrm{P}) \mathrm{H}$ can be achieved via catalysis, but the unequivocal determination of the species involved has yet been fully realized. The current analytical methods of reactant/product analysis based on UV-vis spectroscopy, enzymatic assays, NMR spectroscopy, and HPLC are critically examined here with suggestions for future development.
\end{abstract}

Keywords: Cofactor regeneration; analytical methods; conversion; selectivity; mass balance. 
Reduced Nicotinamide Adenine Dinucleotide (NAD $(P) H)$ is a critical reductant in biochemistry, participating in numerous biological transformations (Box 1). Regeneration of $\mathrm{NAD}(\mathrm{P}) \mathrm{H}$ (i.e., the reduction of $\mathrm{NAD}(\mathrm{P})^{+}$to $\left.\mathrm{NAD}(\mathrm{P}) \mathrm{H}\right)$ has thus far taken on six different forms: enzymatic, chemical, electrocatalytic, photocatalytic, homogeneous, and heterogeneous regeneration, and is a topic of regular reviews.

Albeit a topic of significant recent research, thorough and unequivocal quantification of 1,4$\mathrm{NAD}(\mathrm{P}) \mathrm{H}$ and other products has not been fully achieved. Upon the conversion of $\mathrm{NAD}(\mathrm{P})^{+}$ to $\mathrm{NAD}(\mathrm{P}) \mathrm{H}$, four possible isomers may be formed: 1,2-; 1,4-; and 1,6-NAD(P)H; and the $N A D_{2}$ dimers, with the former three suffering from possible further decay and 1,4-NAD(P)H being the only enzymatically active isomer (i.e., the target product, Figure I). All these isomers contain chromophores and exhibit $\lambda_{\max }$ at $395,340,345$, and $\sim 340 \mathrm{~nm}$ respectively, whereas their common adenine group exhibits $\lambda_{\max }$ at $260 \mathrm{~nm}$ (Figure I). Considering this, it is surprising that a significant proportion of literature uses ultraviolet-visible (UV-vis) spectroscopy to track the absorbance at $340 \mathrm{~nm}$ solely (Figure $1 \mathrm{~A}$, or sometimes the ratio of the absorbance at $260 \mathrm{~nm}$ and $340 \mathrm{~nm}$ [5]) to quantify the concentration of 1,4-NAD(P)H [7]. Using the $\lambda_{\max }$ at $340 \mathrm{~nm}$ and applying the molar absorption coefficient for $1,4-\mathrm{NAD}(\mathrm{P}) \mathrm{H}$ will undoubtedly lead to erroneous results when the reaction is unselective. Specifically, the molar absorption coefficients for 1,4-, 1,6- and the dimer are 6.22, 6.5, and 6.5-7.8 L mol-1 $\mathrm{cm}^{-1}$ [8], respectively, so the activity (and selectivity) of the regeneration system will be overestimated. Given the booming interest and effort in this field [3-12], it is crucial that the concentrations of the products and unreacted reactant are unambiguously quantified.

In addition to using UV-vis spectroscopy, it is also fairly common practice to use a biological assay. A direct assay typically consists of a substrate (e.g., acetaldehyde or $\mathrm{CO}_{2}$ ), a sample (the NAD mixture from a reaction) and an enzyme (e.g., alcohol dehydrogenase (ADH) or formate dehydrogenase $(F D H)$ ) that only utilizes the enzymatically active 1,4-NAD(P)H (Figure 1B). Tracking the initial absorbance at $340 \mathrm{~nm}$ and monitoring the consumption as the reaction progresses (typically in comparison with a commercial 1,4-NAD(P)H sample) will determine the concentration of $1,4-\mathrm{NAD}(\mathrm{P}) \mathrm{H}$ in the system (Figure $1 \mathrm{C}$ ) $[3,9]$. Although using a combination of UV-vis spectroscopy and biological assays is an improvement on relying on UV-vis spectroscopy alone, it only correctly measures the amount of 1,4-NAD(P)H formed and 
still does not provide a complete picture of what is occurring when other products are being generated. Often, authors tend to speculatively suggest some isomers have been formed, but this has not clarified the reaction mechanism or helped to develop better catalytic materials.

A further improvement to using a direct biological assay with UV-vis spectroscopy is using combined biological assays. Unlike a direct biological assay, a cyclic assay makes use of two enzymes and two substrates, catalyzing the reduction of one substrate (e.g., 3-(4,5dimethylthiazol-2-yl)-2,5-diphenyltetrazolium bromide (MTT)) using 1,4-NADH and oxidizing the other substrate (e.g., lactate) using NAD+ (Figure 1D). Tracking the formation of one of the products will give the total concentration of $1,4-\mathrm{NADH}$ and $\mathrm{NAD}^{+}$in the system (Figure $1 \mathrm{E})$. Using this idea, we have recently developed an analytical method that can track NAD+ (for conversion determination) and the monomers of NADH using a combination of UV-vis spectroscopy (340, 395 and $565 \mathrm{~nm}$ ) and two biological assays (for 1,4-NADH and the total of 1,4-NADH and $\mathrm{NAD}^{+}$) [7]. Although this is a facile and relatively easy method to perform in the laboratory, it cannot examine the potential formation of $\mathrm{NAD}_{2}$ dimers.

The use of ${ }^{1} \mathrm{H}$ NMR to assess the catalytic performance of a regeneration method has been used to varying degrees of success. A combination of UV-vis spectroscopy, direct biological assays, and ${ }^{1} \mathrm{H} N M R$ has been used to deduce the activity and selectivity towards $1,4-\mathrm{NAD}(\mathrm{P}) \mathrm{H}$. Typically, the absorbance at $340 \mathrm{~nm}$ is used to calculate an 1,4-NAD(P)H concentration, a direct assay is used to determine the enzymatically active component, and ${ }^{1} \mathrm{H} N M R$ is used to show the appearance of a peak at $6.96 \mathrm{ppm}$ which is characteristic of 1,4-NADH (Figure 1F) [10]. It would be reasonable then to claim that this regeneration system is fully selective; however, this evidence is not fully conclusive. Because $100 \%$ conversion is frequently not achieved (i.e., the mass balance is not closed), it cannot be said with complete certainty that other products (e.g., the enzymatically inactive isomer 1,2-NAD(P)H) have not been formed. This is because the concentrations of reactant used in the literature are typically very low $<<$ $1 \mathrm{mM}$ ) and ${ }^{1} \mathrm{H}$ NMR signals are rather weak for potential side product formation (with even lower concentrations) and the majority of publications show only selectively part of the spectrum, where a full picture could not be assessed. Moreover, 1,2-NAD(P)H is known for its instability, especially in $\mathrm{pH} \sim 7$ phosphate buffers in which most reactions are performed in. So it is possible that this isomer could be formed and would go undetected by the 
quantification method. Similarly, misleading results may be obtained using only the integrals (I) of the signals at $9.33 \mathrm{ppm}\left(\mathrm{NAD}^{+}\right)$and $6.96 \mathrm{ppm}(1,4-\mathrm{NADH})$ according to Yield $(\%)=$ $\mathrm{I}_{6.96} /\left(\mathrm{I}_{6.96}+\mathrm{l}_{9.33}\right) \times 100 \%[11]$.

There have been some attempts on using high-performance liquid chromatography (HPLC) to monitor the progress of the regeneration reaction. However, to the best of our knowledge, it has thus far been limited to the quantification of $\mathrm{NAD}(\mathrm{P})^{+}$and $\mathrm{NAD}(\mathrm{P}) \mathrm{H}$ generally, and not the specific isomers. For example, some authors have used HPLC in combination with UV-vis spectroscopy to track the formation of $\mathrm{NAD}(\mathrm{P}) \mathrm{H}$ via the increase in the absorbance at $340 \mathrm{~nm}$ (UV-vis spectroscopy) and the conversion of $\mathrm{NAD}^{+}$by the change in the ratio of $\mathrm{NAD}^{+} / \mathrm{NADH}$ (HPLC) [12]. Although this method provides a good indication of the conversion of the reaction by means of tracking the reactant consumption, it still does not reveal the true selectivity of the regeneration method. Beaupre and colleagues reported an HPLC method that could separate and quantify both $\mathrm{NAD}^{+}$and 1,2-, 1,4- and 1,6-NADH (Figure 1G) [8]. Although the paper is not investigating the regeneration of $1,4-\mathrm{NAD}(\mathrm{P}) \mathrm{H}$, it shows the feasibility of using HPLC to separate and quantify the different NADH isomers, albeit except for the dimers, which was not attempted. From a reaction engineering perspective, mass/materials balance is of great importance in studying any reactive systems. Without such fundamental information, it would be impossible to understand reaction mechanisms and design better catalysts. Although combined UV-vis spectroscopy and biological assays can realize the same level of product qualifications as the use of HPLC (i.e., dimers not detected), we suggest HPLC be used as a routine tool for this particular application as it allows more sensitive detection of potential by-product formation and room for method development.

In summary, the dependence on UV-vis spectroscopy alone affords inaccurate and often misleading results. As such, it is not a good descriptor of catalytic performance of the NAD(P)H regeneration systems. The use of ${ }^{1} \mathrm{H} N M R$ together with UV-vis spectroscopy provides a clearer picture still, allowing the identification of the unselective isomers, but as of now, quantification is still unattainable. Combining UV-vis spectroscopy and two biological assays or the use of HPLC is the most thorough and unequivocal form of analysis, providing quantitative analysis of both reactant, desired product, and by-products. Although both procedures have yet involved the quantification of any dimeric species, it is hoped that in the 
near future with the help of analytical chemists and engineers, all species can be fully accounted for to reveal comprehensive reaction paths for the design of more efficient and cleaner strategies of cofactor regeneration for sustainable biosynthesis.

\section{Acknowledgements}

This line of research in The Wang Group has been developed with financial support from The Royal Society (ICA\R1\180317, IES\R3\170162, IE150611 and RG150001), The EPSRC UK Catalysis Hub (via Energy Theme), British Council Newton Fund and The Carnegie Trust for the Universities of Scotland (70265). We thank Dr. Tony Saba, Mr. Jianwei Li, Dr. Wilm Jones, Dr. Panagiotis N. Kechagiopoulos and Prof. James A. Anderson for their contribution to these projects.

\section{References}

1. Sheldon, R. and Brady, D. (2019) Broadening the scope of biocatalysis in sustainable organic synthesis. ChemSusChem. 12, 2859-2881.

2. Li, Z. et al. (2019) Production of value-added chemicals from glycerol using in vitro enzymatic cascades. Commun. Chem. 1, 71.

3. Zhang, S. et al. (2019) Artificial thylakoid for the coordinated photoenzymatic reduction of carbon dioxide. ACS Catal. 9, 3913-3925.

4. Soldevila-Barreda, J.J. et al. (2015) Transfer hydrogenation catalysis in cells as a new approach to anticancer drug design. Nat. Commun. 6, 6582.

5. Zor, C. et al. (2017) $\mathrm{H}_{2}$-Driven biocatalytic hydrogenation in continuous flow using enzymemodified carbon nanotube columns. Chem. Commun. 53, 9839-9841.

6. Kuk, S. et al. (2017) Photoelectrochemical reduction of carbon dioxide to methanol through a highly efficient enzyme cascade. Angew. Chem. Int. Ed. 56, 3827-3832.

7. Saba, T. et al. (2020). A facile analytical method for reliable selectivity examination in cofactor NADH regeneration. Chem. Commun. 56, 1231-1234.

8. Beaupre, B. et al. (2015) Metabolic function for human renalase: oxidation of isomeric forms of $\beta-N A D(P) H$ that are inhibitory to primary metabolism. Biochem. 3, 795-806.

9. Brown, K. et al. (2016) Photocatalytic regeneration of nicotinamide cofactors by quantum dot-enzyme biohybrid complexes. ACS Catal. 6, 2201-2204.

10. Yuan, M. et al. (2019) Efficient NADH regeneration by a redox polymer-immobilized 
enzymatic system. ACS Catal. 9, 5486-5495.

11. Wang, X. and Yiu, H.H.P. (2016) Heterogeneous catalysis mediated cofactor NADH regeneration for enzymatic reduction. ACS Catal. 6, 1880-1886.

12. Tang, L. et al. (2015) Activation of dioxygen by a TAML activator in reverse micelles: characterization of an Fe ${ }^{I I I} \mathrm{Fe}^{\mathrm{IV}}$ dimer and associated catalytic chemistry. J. Am. Chem. Soc. 30, 9704-9715. 
Box 1. Biocatalytic transformations and the necessity of NAD(P)H regeneration.

Biocatalysis is playing an increasingly important role industrially, capable of performing complex chemical transformations under relatively benign reaction conditions. Due to their inherently high activity, selectivity, and specificity, enzymes are ideal candidates for a wide variety of reactions [1]. Among the enzymes used in biosynthesis, oxidoreductases are the largest class of commercially applied enzymes and are capable of performing a vast array of oxidative and reductive chemical transformations [1], involving the production of chiral intermediates, amino acids, steroids, and other pharmaceutically important chemicals, as well as the fixation and subsequent conversion of molecules such as $\mathrm{CO}_{2}$ [2-6]. These transformations are dependent on $\mathrm{NAD}(\mathrm{P}) \mathrm{H}$, a cofactor that acts as a source of hydride and is subsequently oxidised to $\mathrm{NAD}(\mathrm{P})^{+}$during the reaction (Figure I). Unlike the enzymes themselves, the cofactor $\mathrm{NAD}(\mathrm{P}) \mathrm{H}$ is stoichiometrically consumed and due to its high cost $(\$ 2600 / \mathrm{mol})$, regeneration of the cofactor is necessary for its practical use [7].

(A)

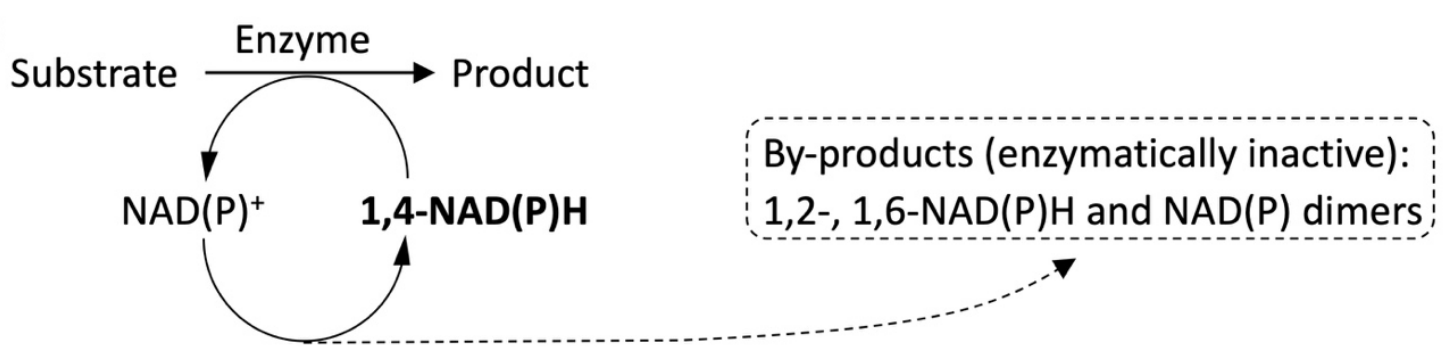

(B)

$$
\text { Regeneration }
$$
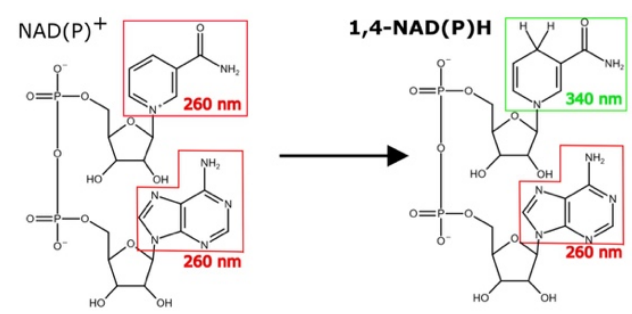

NAD(P) Dimer

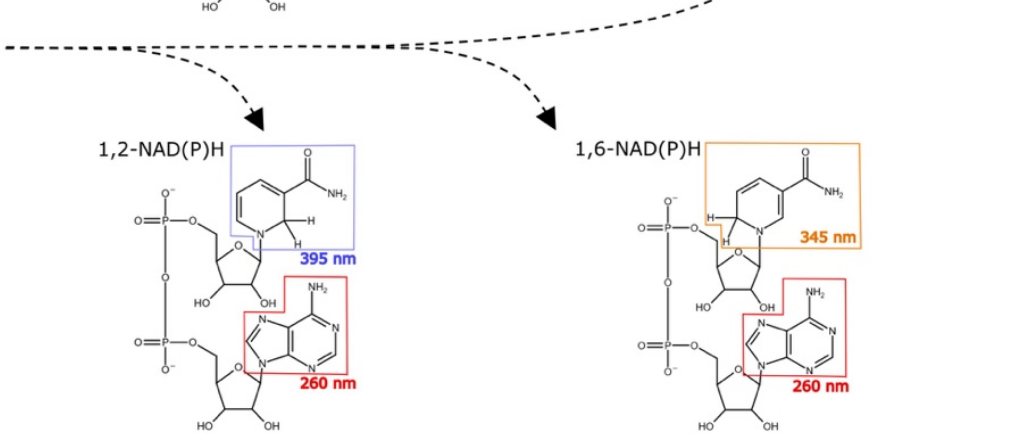

Figure I. Schematic representation of enzymatic reaction using cofactor 1,4-NAD(P)H with in situ regeneration. (A) Enzymatic reduction consuming 1,4-NAD $(\mathrm{P}) \mathrm{H}$ and its regeneration to various products. (B) Structures of $\mathrm{NAD}(\mathrm{P}) \mathrm{H}$ isomers and their associated absorption maxima, $\mathrm{R}=$ adenosine diphosphoribose. 
(A)

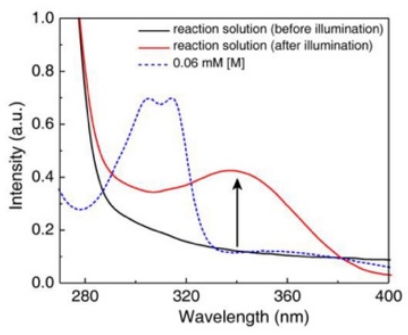

(B)

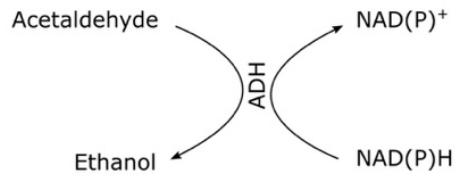

(D)

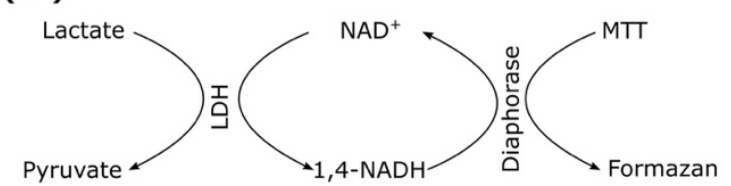

$(\mathrm{F})$
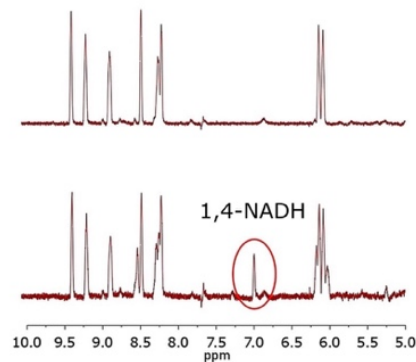

(G)

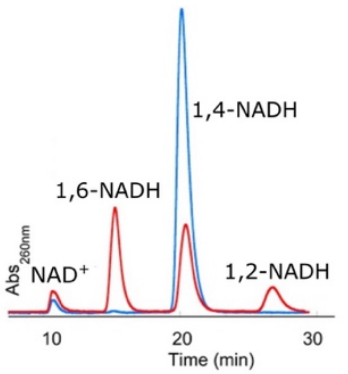

(C)

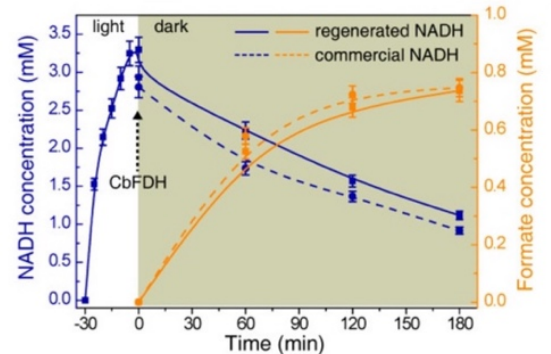

(E)

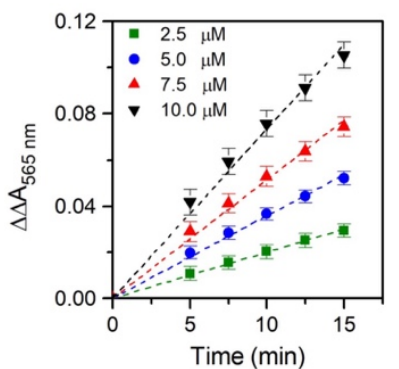

Figure 1. The analytical methods currently used for 1,4-NADH quantification. (A) Representative spectra showing the increase in the UV-vis spectroscopy absorbance at $340 \mathrm{~nm}$ as the reaction progresses, $\mathrm{M},\left[\mathrm{Cp}^{*} \mathrm{Rh} \text {-(bpy) } \mathrm{H}_{2} \mathrm{O}\right]^{2+}\left(\mathrm{Cp}^{*}=\right.$ pentamethylcyclopentadienyl, bpy $=2,2^{\prime}$-bipyridyl), is a typical organic mediator used in photocatalytic/electrocatalytic regeneration. Adapted with permission from [3]. (B) An example of a direct biological assay. (C) Comparison of commercial 1,4$\mathrm{NADH}$ and regenerated NADH when used in a direct biological assay (involving $\mathrm{CO}_{2}$ to formate reaction, CbFDH: FDH from Candida boidinii). Adapted with permission from [3]. (D) An example of a cyclic biological assay (LDH: lactate dehydrogenase). (E) A time on stream profile showing the increase in the absorbance at $565 \mathrm{~nm}$, corresponding to formation of formazan in a biological cyclic assay, with different total concentrations of 1,4-NADH and NAD ${ }^{+}$. Adapted with permission from [7]. (F) ${ }^{1} \mathrm{H}$ NMR profile showing the formation of 1,4-NADH. Adapted with permission from [10]. (G) The separation of $\mathrm{NAD}^{+}$and the monomeric isomers of 1,4-NADH in HPLC. Adapted with permission from [8]. 